\title{
TITLE:
}

\section{A weighted independent even factor algorithm}

AUTHOR(S):

Takazawa, Kenjiro

\section{CITATION:}

Takazawa, Kenjiro. A weighted independent even factor algorithm. Mathematical Programming 2012, 132(1-2): 261-276

ISSUE DATE:

2012-04

URL:

http://hdl.handle.net/2433/154867

\section{RIGHT:}

The final publication is available at www.springerlink.com; This is not the published version. Please cite only the published version.; この論文 は出版社版でありません。引用の際には出版社版をご確認ご利用くだ さい。 


\title{
A weighted independent even factor algorithm
}

\author{
Kenjiro Takazawa \\ Research Institute for Mathematical Sciences, \\ Kyoto University, Kyoto 606-8502, Japan. \\ takazawa@kurims.kyoto-u.ac.jp
}

\begin{abstract}
An even factor in a digraph is a vertex-disjoint collection of directed cycles of even length and directed paths. An even factor is called independent if it satisfies a certain matroid constraint. The problem of finding an independent even factor of maximum size is a common generalization of the nonbipartite matching and matroid intersection problems.

In this paper, we present a primal-dual algorithm for the weighted independent even factor problem in odd-cycle-symmetric weighted digraphs. Cunningham and Geelen have shown that this problem is solvable via valuated matroid intersection. Their method yields a combinatorial algorithm running in $\mathrm{O}\left(n^{3} \gamma+n^{6} m\right)$ time, where $n$ and $m$ are the number of vertices and edges, respectively, and $\gamma$ is the time for an independence test. In contrast, combining the weighted even factor and independent even factor algorithms, our algorithm works more directly and runs in $\mathrm{O}\left(n^{4} \gamma+n^{5}\right)$ time. The algorithm is fully combinatorial, and thus provides a new dual integrality theorem which commonly extends the total dual integrality theorems for matching and matroid intersection.
\end{abstract}

Keywords: Independent even factor, Combinatorial algorithm, Dual integrality, Nonbipartite matching, Matroid intersection

Mathematics Subject Classification (2000): 90C27, 05C70, 52B40

\section{Introduction}

The nonbipartite matching and matroid intersection problems are two fundamental problems which can be solved efficiently. Elegant results such as combinatorial algorithms $[6,8,14]$ and totally dual integral (TDI) description $[4,7]$ have been known for a long time. As a common generalization of these two problems, Cunningham and Geelen [3] introduced the independent path-matching problem and showed a min-max formula, a TDI description and a polynomial algorithm based on the ellipsoid method. Then, combinatorial approaches to path-matchings followed [9, 22, 23].

In this context, Cunningham and Geelen introduced a further generalization, independent even factors, in their unpublished manuscript in 2001 (see also Cunningham's paper [2]). Let $(G, c)$ be a weighted digraph with $G=(V, E)$ and $c \in \mathbf{R}_{+}^{E}$, and let $\mathbf{M}^{+}$and $\mathbf{M}^{-}$be two matroids on $V$. An edge set $M \subseteq E$ is an even factor in $G$ if $M$ forms a vertex-disjoint collection of directed cycles of even length and directed paths. (A path may have odd length.) An even factor $M$ is independent if the set of vertices which have a leaving edge in $M$ is independent in $\mathbf{M}^{+}$and the set of vertices which have an entering edge in $M$ is independent in $\mathbf{M}^{-}$. A related optimization problem is to find an (independent) even factor maximizing $|M|$, or maximizing $c(M)=\sum_{e \in M} c(e)$ in the weighted version. That is, we have four versions: the even factor problem (EFP); the weighted even factor 
problem (WEFP); the independent even factor problem (IEFP); and the weighted independent even factor problem (WIEFP).

In the manuscript, Cunningham and Geelen exhibited that the EFP is NP-hard in general digraphs and polynomially solvable in weakly symmetric digraphs, in which every edge $e$ in any directed cycle has the reverse edge $\bar{e}$. As for the WEFP, they considered weakly symmetric weighted digraphs. A weighted digraph $(G, c)$ is weakly symmetric if $G$ is weakly symmetric and $c(e)=c(\bar{e})$ if $e, \bar{e} \in E$. They proposed a linear programming description of the even factors in weakly symmetric weighted digraphs which has dual integrality. They also proposed a primal-dual method for solving the WEFP which calls an algorithm for the EFP polynomially many times.

Cunningham and Geelen also showed a reduction of the IEFP in weakly symmetric digraphs to matroid intersection, which calls an algorithm for the EFP polynomially many times. Also, they reduced the WIEFP in weakly symmetric weighted digraphs to valuated matroid intersection $[15$, 16], which calls an algorithm for the WEFP polynomially many times.

We remark here that the class of weakly symmetric weighted digraphs is broad enough to include the matching and matroid intersection problems. Further, Cunningham and Geelen's approach applies to a broader class of digraphs, called odd-cycle-symmetric. A digraph is odd-cycle-symmetric if every directed cycle of odd length (odd cycle) $C$ has the reverse directed cycle $\bar{C}$. A weighted digraph $(G, c)$ is odd-cycle-symmetric if $G$ is odd-cycle-symmetric and $c(C)=c(\bar{C})$ for every odd cycle $C$. Note that a weakly symmetric (weighted) digraph is odd-cycle-symmetric.

Several important theorems of nonbipartite matching are extended to even factors in oddcycle-symmetric digraphs. The Tutte-Berge formula and the Edmonds-Gallai decomposition are extended by Cunningham and Geelen, and Pap and Szegö [21]. Király and Makai [12] presented a linear description of even factors in odd-cycle-symmetric weighted digraphs and proved its dual integrality, which corresponds to the TDI description for matching. Harvey's algebraic matching algorithm [10] applies to the IEFP in an odd-cycle-symmetric digraph with two matroids linearly represented over the same field.

Properties of odd-cycle-symmetric digraphs are also studied. A characterization of odd-cyclesymmetric digraphs is given by Z. Király (see [12]). Kobayashi and Takazawa [13] showed that the odd-cycle-symmetry of a digraph is a necessary and sufficient condition for the degree sequences of the even factors in the digraph to form a jump system [1], and the odd-cycle-symmetry of a weighted digraph is also a necessary and sufficient condition for the weighted even factors to induce an M-concave function on the jump system [17]. This implies that the odd-cycle-symmetry is a natural assumption in considering optimization problems involving even factors.

A main interest for even factors had been whether we can design a combinatorial algorithm for the EFP in odd-cycle-symmetric digraphs. This had been open for several years since the introduction of even factors, and was solved by Pap [20]. He presented an augmenting path algorithm similar to Edmonds' matching algorithm [6]. Recently, Takazawa [24] extended Pap's algorithm to the WEFP by combining it with the weighted matching algorithm [5]. Also, Iwata and Takazawa [11] extended Pap's algorithm to the IEFP by combining it with the matroid intersection algorithms $[8,14]$.

The contribution of this paper is a combinatorial algorithm for the WIEFP in odd-cyclesymmetric weighted digraphs. By calling the algorithm for the WEFP [24] in the valuated matroid intersection algorithm [16], Cunningham and Geelen's method also achieves a combinatorial algorithm for the WIEFP running in $\mathrm{O}\left(n^{3} \gamma+n^{6} m\right)$ time, where $n$ and $m$ are the number of vertices and edges, respectively, and $\gamma$ is the time for an independence test. On the other hand, our algorithm combines the algorithms for the WEFP [24] and IEFP [11]. In other words, the algorithm commonly extends classical algorithms for the weighted matching and weighted matroid intersection 
problems, and works directly on the WIEFP. The time complexity of our algorithm is $\mathrm{O}\left(n^{4} \gamma+n^{5}\right)$, which is better if $\gamma=\mathrm{O}\left(n^{2} m\right)$.

The algorithm finds an integer optimal solution for a linear program corresponding to the WIEFP, and simultaneously finds an integer optimal solution for the dual program if the weight is integer. Thus, it provides a new dual integrality theorem (Theorem 4) which commonly extends those for matching, matroid intersection, independent path-matching and even factors $[3,4,7,12]$.

This paper is organized as follows. Section 2 provides a formal definition of the WIEFP. In Section 3, we show a linear program that corresponds to the WIEFP and two matroid operations used in our algorithm. Algorithm description appears in Section 4.

\section{Definitions}

\subsection{Basic notations}

Let $G=(V, E)$ be a digraph with vertex set $V$ and edge set $E$. We denote an edge $e$ from $u$ to $v$ by $u v$. The reverse edge of $e$ is denoted by $\bar{e}$. The initial vertex and terminal vertex of $e$ are respectively denoted by $\partial^{+} e$ and $\partial^{-} e$, i.e., $\partial^{+} e=u$ and $\partial^{-} e=v$. Similarly, for $F \subseteq E$, define $\partial^{+} F=\left\{\partial^{+} e \mid e \in F\right\}$ and $\partial^{-} F=\left\{\partial^{-} e \mid e \in F\right\}$. For $U \subseteq V$, let $\delta^{+} U=\left\{e \mid e \in E, \partial^{+} e \in U\right\}$ and $\delta^{-} U=\left\{e \mid e \in E, \partial^{-} e \in U\right\}$. Denote $E[U]=\left\{e \mid e \in E, \partial^{+} e, \partial^{-} e \in U\right\}$. For $x \in \mathbf{R}^{E}$ and $F \subseteq E$, denote $x(F)=\sum_{e \in F} x(e)$. For two sets $F_{1}$ and $F_{2}$, their symmetric difference $\left(F_{1} \backslash F_{2}\right) \cup\left(F_{2} \backslash F_{1}\right)$ is denoted by $F_{1} \triangle F_{2}$.

A subset of edges $\left\{e_{1}, \ldots, e_{k}\right\}$ is said to be a path if $\partial^{+} e_{1}, \partial^{-} e_{1}=\partial^{+} e_{2}, \partial^{-} e_{2}=\partial^{+} e_{3}$, $\ldots, \partial^{-} e_{k-1}=\partial^{+} e_{k}$, and $\partial^{-} e_{k}$ are distinct. A cycle is a subset of edges $\left\{e_{1}, \ldots, e_{k}\right\}$ such that $\partial^{-} e_{1}=\partial^{+} e_{2}, \partial^{-} e_{2}=\partial^{+} e_{3}, \ldots, \partial^{-} e_{k-1}=\partial^{+} e_{k}$ and $\partial^{-} e_{k}=\partial^{+} e_{1}$ are distinct. For a cycle $C=\left\{e_{1}, \ldots, e_{k}\right\}$, the reverse cycle $\bar{C}$ of $C$ is $\left\{\bar{e}_{1}, \ldots, \bar{e}_{k}\right\}$. A path or a cycle $F=\left\{e_{1}, \ldots, e_{k}\right\}$ is said to be odd if $k$ is odd, and even if $k$ is even. For $F, V(F)$ denotes the set of incident vertices $\bigcup_{i=1}^{k}\left\{\partial^{+} e_{i}, \partial^{-} e_{i}\right\}$.

In this paper, we indicate a matroid by the pair of its ground set and independent set family, say, $\mathbf{M}=(V, \mathcal{I})$. We expect the readers to be familiar with basic concepts in matroid theory [18], such as the rank function $\rho$, the closure function $\mathrm{cl}$ and a fundamental circuit with respect to $I \in \mathcal{I}$ and $v \in \operatorname{cl}(I) \backslash I$, denoted by $C(I \mid v)$.

Let $\mathbf{M}=(V, \mathcal{I})$ and $\mathbf{M}^{\prime}=\left(V^{\prime}, \mathcal{I}^{\prime}\right)$ be matroids such that $V \cap V^{\prime}=\emptyset$. We denote the direct sum of $\mathbf{M}$ and $\mathbf{M}^{\prime}$ by $\mathbf{M} \oplus \mathbf{M}^{\prime}$. For $U \subseteq V$, the restriction of $\mathbf{M}$ to $U$ is denoted by $\mathbf{M} \mid U$, and the contraction of $U$ from $\mathbf{M}$ is denoted by $\mathbf{M} / U$. That is, the independent set families of $\mathbf{M} \oplus \mathbf{M}^{\prime}, \mathbf{M} \mid U$, and $\mathbf{M} / U$ are $\left\{I \cup I^{\prime} \mid I \in \mathcal{I}, I^{\prime} \in \mathcal{I}^{\prime}\right\},\{I \mid I \subseteq U, I \in \mathcal{I}\}$, and $\left\{I \mid I \subseteq V \backslash U, I \cup B_{U} \in \mathcal{I}\right.$ for some base $B_{U}$ of $\left.(\mathbf{M} \mid U)\right\}$, respectively.

\section{$2.2 \quad$ Problem definition}

Let $(G, c)$ be a weighted digraph with $G=(V, E)$ and $c \in \mathbf{R}_{+}^{E}$. Also, let $\mathbf{M}^{+}=\left(V, \mathcal{I}^{+}\right)$and $\mathbf{M}^{-}=\left(V, \mathcal{I}^{-}\right)$be matroids. The following is the definition of the central object in this paper.

Definition 1 (Independent even factors). A subset of edges $M \subseteq E$ is an even factor in $G$ if it forms a vertex-disjoint collection of paths and even cycles. Moreover, $M$ is said to be an independent even factor in $\left(G, \mathbf{M}^{+}, \mathbf{M}^{-}\right)$if $M$ is an even factor with $\partial^{+} M \in \mathcal{I}^{+}$and $\partial^{-} M \in \mathcal{I}^{-}$.

The topic of this paper is the weighted independent even factor problem (WIEFP), defined as follows. 
Problem 2 (WIEFP). For a weighted digraph $(G, c)$ and matroids $\mathbf{M}^{+}, \mathbf{M}^{-}$, find an independent even factor $M$ in $\left(G, \mathbf{M}^{+}, \mathbf{M}^{-}\right)$that maximizes $c(M)$.

The weighted nonbipartite matching problem and the weighted matroid intersection problem are special cases of the WIEFP.

Weighted matching. Let $(\bar{G}, \bar{c})$ be a weighted undirected graph with $\bar{G}=(V, \bar{E})$ and $\bar{c} \in \mathbf{R}_{+}^{\bar{E}}$ in which you are supposed to find a maximum-weight matching. Then, construct an instance $\left(G, c, \mathbf{M}^{+}, \mathbf{M}^{-}\right)$of the WIEFP as follows: $G=(V, E)$, where $E=\{u v, v u \mid u, v \in V$ are adjacent in $\bar{G}\}$; $c(u v)=\bar{c}(\{u, v\})$, where $\{u, v\} \in \bar{E}$ is an edge connecting $u$ and $v$; and both $\mathbf{M}^{+}$and $\mathbf{M}^{-}$are free. Observe that in $\left(G, c, \mathbf{M}^{+}, \mathbf{M}^{-}\right)$there exists a maximum-weight independent even factor consisting of even cycles. By alternately picking up edges along these cycles, we obtain a vertex-disjoint set of edges, which corresponds to a maximum-weight matching in $(\bar{G}, \bar{c})$.

Weighted matroid intersection. Let $\mathbf{M}_{1}=\left(V, \mathcal{I}_{1}\right)$ and $\mathbf{M}_{2}=\left(V, \mathcal{I}_{2}\right)$ be matroids and let $\bar{c} \in \mathbf{R}_{+}^{V}$ be a weight vector. Then, construct an instance $\left(G, c, \mathbf{M}^{+}, \mathbf{M}^{-}\right)$of the WIEFP as follows. Let $V^{+}=\left\{v^{+} \mid v \in V\right\}$ and $V^{-}=\left\{v^{-} \mid v \in V\right\}$ be copies of $V$. For each $U \subseteq V$, we denote the corresponding copies in $V^{+}$and $V^{-}$by $U^{+}$and $U^{-}$, respectively. Let $G=\left(V^{+} \cup V^{-}, E\right)$, where $E=\left\{v^{+} v^{-} \mid v \in V\right\}$, and $c\left(v^{+} v^{-}\right)=\bar{c}(v)$. Attach two matroids $\mathbf{M}^{+}=\left(V^{+} \cup V^{-}, \mathcal{I}^{+}\right)$and $\mathbf{M}^{-}=$ $\left(V^{+} \cup V^{-}, \mathcal{I}^{-}\right)$, where $\mathcal{I}^{+}=\left\{U^{+} \mid U \in \mathcal{I}_{1}\right\}$ and $\mathcal{I}^{-}=\left\{U^{-} \mid U \in \mathcal{I}_{2}\right\}$. If $M=\left\{v^{+} v^{-} \mid v \in U \subseteq V\right\}$ is an independent even factor in $\left(G, \mathbf{M}^{+}, \mathbf{M}^{-}\right)$maximizing $c(M)$, then $U$ is a common independent set in $\mathbf{M}_{1}$ and $\mathbf{M}_{2}$ maximizing $\bar{c}(U)$.

The WIEFP is NP-hard even for the special case of the EFP, namely, $c \in\{0,1\}^{E}$ and both $\mathbf{M}^{+}$ and $\mathbf{M}^{-}$are free. In order to make the problem tractable, we assume the following property for the given weighted digraph.

Definition 3 (Odd-cycle-symmetric weighted digraphs). A digraph $G$ is odd-cycle-symmetric if every odd cycle $C$ in $G$, the reverse cycle $\bar{C}$ is in $G$. A weighted digraph $(G, c)$ is odd-cyclesymmetric if $G$ is odd-cycle-symmetric and $c(C)=c(\bar{C})$ for every odd cycle $C$.

In the following sections, we deal with the WIEFP in odd-cycle-symmetric weighted digraphs with general matroids. Note that, by the above reductions, the class of odd-cycle-symmetric weighted digraphs is broad enough to include the weighted matching and weighted matroid intersection problems.

\section{Preliminaries for the algorithm}

\subsection{Linear programming formulation}

Let $\left(G, c, \mathbf{M}^{+}, \mathbf{M}^{-}\right)$be an instance of the WIEFP, where $G=(V, E), c \in \mathbf{R}_{+}^{E}, \mathbf{M}^{+}=\left(V, \mathcal{I}^{+}\right)$ and $\mathbf{M}^{-}=\left(V, \mathcal{I}^{-}\right)$. Assume that $(G, c)$ is odd-cycle-symmetric. The rank function of $\mathbf{M}^{+}$and $\mathbf{M}^{-}$is denoted by $\rho^{+}$and $\rho^{-}$, respectively. Similarly, objects of $\mathbf{M}^{+}$and $\mathbf{M}^{-}$are denoted with superscript + and - , respectively, e.g. $\mathrm{cl}^{+}(I), C^{+}(I \mid v), \mathrm{cl}^{-}(I)$ and $C^{-}(I \mid v)$.

As an extension of the linear description of the related problems, we consider the following 
linear program, where $\mathcal{U}=\{U|U \subseteq V| U \mid, \geq 3$ is odd $\}$ :

$$
\begin{array}{cll}
\text { (P) maximize } & \sum_{e \in E} c(e) x(e) & \\
\text { subject to } & x\left(\delta^{+} U\right) \leq \rho^{+}(U) & (U \subseteq V), \\
& x\left(\delta^{-} U\right) \leq \rho^{-}(U) & (U \subseteq V), \\
& x(E[U]) \leq|U|-1 & (U \in \mathcal{U}), \\
& x(e) \geq 0 & (e \in E) .
\end{array}
$$

Note that a characteristic vector of an independent even factor in $\left(G, \mathbf{M}^{+}, \mathbf{M}^{-}\right)$is an integer feasible solution for $(\mathrm{P})$, and vice versa. The dual program of $(\mathrm{P})$ is given by

$$
\begin{aligned}
\text { (D) minimize } & \sum_{U \subseteq V}\left(\rho^{+}(U) y^{+}(U)+\rho^{-}(U) y^{-}(U)\right)+\sum_{U \in \mathcal{U}}(|U|-1) z(U) \\
\text { subject to } & \sum_{\substack{U \subseteq V, U \ni \partial^{+} e}} y^{+}(U)+\sum_{\substack{U \subseteq V, U \ni \partial^{-}}} y^{-}(U)+\sum_{\substack{U \in \mathcal{U}, e \in E[U]}} z(U) \geq c(e) \quad(e \in E), \\
& y^{+}(U) \geq 0 \quad(U \subseteq V), \\
& y^{-}(U) \geq 0 \quad(U \subseteq V), \\
& z(U) \geq 0 \quad(U \in \mathcal{U}) .
\end{aligned}
$$

Denote

$$
c^{\prime}(e)=\sum_{U \ni \partial^{+} e} y^{+}(U)+\sum_{U \ni \partial^{-} e} y^{-}(U)+\sum_{U \in \mathcal{U}: e \in E[U]} z(U)-c(e) .
$$

The constraint (1) is rewritten as

$$
c^{\prime}(e) \geq 0 \quad(e \in E)
$$

and the complementary slackness (CS) conditions are

$$
\begin{aligned}
x(e)>0 & \Longrightarrow c^{\prime}(e)=0, \\
y^{+}(U)>0 & \Longrightarrow x\left(\delta^{+} U\right)=\rho^{+}(U), \\
y^{-}(U)>0 & \Longrightarrow x\left(\delta^{-} U\right)=\rho^{-}(U), \\
z(U)>0 & \Longrightarrow x(E[U])=|U|-1 .
\end{aligned}
$$

In Section 4, we will present an algorithm for finding optimal solutions for (P) and (D), in other words, feasible solutions for (P) and (D) satisfying (6)-(9). In particular, the algorithm finds an integer optimal solution for $(\mathrm{P})$, which is the characteristic vector of a maximum-weight independent even factor. Also, the algorithm finds an optimal solution for (D) when $c$ is integer. Thus, the algorithm constructively proves the following integrality theorem, which corresponds to the TDI theorems for matching [4] and matroid intersection [8]. We say that a set family $\mathcal{F}$ is laminar if $U_{1} \subseteq U_{2}, U_{2} \subseteq U_{1}$ or $U_{1} \cap U_{2}=\emptyset$ for all $U_{1}, U_{2} \in \mathcal{F}$. Also, we say that $\mathcal{F}$ is nested if $U_{1} \subseteq U_{2}$ or $U_{2} \subseteq U_{1}$ for all $U_{1}, U_{2} \in \mathcal{F}$.

Theorem 4. For an instance $\left(G, c, \mathbf{M}^{+}, \mathbf{M}^{-}\right)$of the WIEFP, $(\mathrm{P})$ has an integer optimal solution if $(G, c)$ is odd-cycle-symmetric. Moreover, if $(G, c)$ is odd-cycle-symmetric and $c$ is integer, (D) also has an integral optimal solution $\left(y^{+}, y^{-}, z\right)$ such that $\left\{U \mid y^{+}(U)>0\right\}$ and $\left\{U \mid y^{-}(U)>0\right\}$ are nested and $\{U \mid z(U)>0\}$ is laminar. 


\subsection{Operations on matroids}

This subsection presents two operations on matroids, which play a key role in the algorithm described in Section 4.

\subsubsection{Shrinking of an independent set}

Let $\mathbf{M}=(V, \mathcal{I})$ be a matroid. For an independent set $U \in \mathcal{I}$, construct another matroid as follows. Let $\tilde{V}=(V \backslash U) \cup\{w\}$, where $w$ is a new element, and define a subset family $\tilde{\mathcal{I}}$ of $\tilde{V}$ by

$$
\tilde{\mathcal{I}}=\{I|I \subseteq V \backslash U, \exists J \subseteq U,| J|=| U \mid-1, I \cup J \in \mathcal{I}\} \cup\{I \cup\{w\} \mid I \subseteq V \backslash U, I \cup U \in \mathcal{I}\} .
$$

Then, $(\tilde{V}, \tilde{\mathcal{I}})$ is a matroid, which is referred to as the shrinking of $U$.

Proposition 5 ([11]; see also [19]). The tuple $(\tilde{V}, \tilde{\mathcal{I}})$ forms a matroid.

This operation corresponds to shrinking an odd vertex set in Edmonds' matching algorithm [6] and Pap's even factor algorithm [20]. In the independent even factor algorithm [11], we apply shrinking of $V(C)$ to $\mathbf{M}^{+}$and $\mathbf{M}^{-}$when we shrink an odd cycle $C$, in order to define matroids in the resulting digraph.

\subsection{2 p-minor}

Let $\mathbf{M}=(V, \mathcal{I})$ be a matroid and let $p \in \mathbf{R}_{+}^{V}$. Then, partition $V$ into $V_{1}, V_{2}, \ldots, V_{k}$ so that

$$
V_{i}=\left\{v \mid p(v)=\pi_{i}\right\} \text { for } i=1,2, \ldots, k, \text { where } \pi_{1}>\pi_{2}>\cdots>\pi_{k},
$$

and let $U_{i}=\bigcup_{j=1}^{i} V_{j}$ for $i=1,2, \ldots, k$. Here, define a matroid $\mathbf{M}_{p}$, the $p$-minor of $\mathbf{M}$, by

$$
\mathbf{M}_{p}=\left(\mathbf{M} \mid V_{1}\right) \oplus\left(\left(\mathbf{M} / U_{1}\right) \mid V_{2}\right) \oplus \cdots \oplus\left(\left(\mathbf{M} / U_{k-2}\right) \mid V_{k-1}\right) \oplus\left(\mathbf{M} / U_{k-1}\right) .
$$

The independent set family, the closure function, and a fundamental circuit of $\mathbf{M}_{p}$ are denoted by $\mathcal{I}_{p}, \mathrm{cl}_{p}$, and $C_{p}(\cdot \mid \cdot)$, respectively.

Note that the base family of $\mathbf{M}_{p}$ is exactly a family of bases of $\mathbf{M}$ maximizing $p(\cdot)$. In other words, if $I$ is independent in $\mathbf{M}_{p}$, then there exists a base $B_{I}$ of $\mathbf{M}$ which contains $I$ and maximizes $p(\cdot)$ among all bases of $\mathbf{M}$. Also, the CS conditions (7) and (8) can be restated in terms of $p$-minor of $\mathbf{M}^{+}$and $\mathbf{M}^{-}$, respectively. Given $p$, set

$$
y(U)= \begin{cases}\pi_{i}-\pi_{i+1} & \left(U=U_{i}(i=1, \ldots, k-1)\right), \\ \pi_{k} & (U=V), \\ 0 & \text { (otherwise) }\end{cases}
$$

Then, we have the following proposition.

Proposition 6. For an independent even factor $M$ in $\left(G, \mathbf{M}^{+}, \mathbf{M}^{-}\right)$, the following (i) and (ii) hold:

(i) If $\partial^{+} M \in \mathcal{I}_{p}^{+}$and $p(v)=0$ for $v \in V \backslash \mathrm{cl}_{p}^{+}\left(\partial^{+} M\right)$, then (7) holds for $y^{+}=y$ defined by (10) and (11).

(ii) If $\partial^{-} M \in \mathcal{I}_{p}^{-}$and $p(v)=0$ for $v \in V \backslash \mathrm{cl}_{p}^{-}\left(\partial^{-} M\right)$, then (8) holds for $y^{-}=y$ defined by (10) and (11). 
Proof. We show a proof for (i). Assume the integer $k$ in (10) is at least two, since the case $k=1$ is trivial.

If $\partial^{+} M$ is a base of $\mathbf{M}_{p}^{+}$, then (7) directly follows from the definition of $\mathbf{M}_{p}^{+}$. Suppose $\partial^{+} M$ is not a base of $\mathbf{M}_{p}^{+}$. Since $p(v)=0$ for $v \in V \backslash \mathrm{cl}_{p}^{+}\left(\partial^{+} M\right)$, it holds that $\pi_{k}=0$ and $U_{k-1} \subseteq \mathrm{cl}_{p}^{+}\left(\partial^{+} M\right)$. By the definition of $\mathbf{M}_{p}^{+}$and the fact that $\partial^{+} M$ is independent, we have that $\partial^{+} M \cap U_{i}$ is a base of $\mathbf{M} \mid U_{i}$ for each $i=1, \ldots, k-1$. Thus, $x\left(\delta^{+} U_{i}\right)=\rho^{+}\left(U_{i}\right)$ for $i=1, \ldots, k-1$, and (7) follows.

The same argument proves (ii).

\section{Algorithm description}

Let $(\hat{G}, c)$ be an odd-cycle-symmetric weighted digraph with $\hat{G}=(\hat{V}, \hat{E})$ and $c \in \mathbf{R}_{+}^{\hat{E}}$, and let $\hat{\mathbf{M}}^{+}=\left(\hat{V}, \hat{\mathcal{I}}^{+}\right)$and $\hat{\mathbf{M}}^{-}=\left(\hat{V}, \hat{\mathcal{I}}^{-}\right)$be matroids. The objective of this section is to present an algorithm for finding an independent even factor $\hat{M}$ in $\left(\hat{G}, \hat{\mathbf{M}}^{+}, \hat{\mathbf{M}}^{-}\right)$maximizing $c(\hat{M})$. In what follows, $\hat{\mathbf{M}}^{+}, \hat{\mathbf{M}}^{-}$and $\hat{M}$ represent the objects in the input digraph $\hat{G}$, while $\mathbf{M}^{+}, \mathbf{M}^{-}$and $M$ correspond to the digraph $G=(V, E)$ in process, obtained by applying the procedures of shrinking and expanding of odd cycles repeatedly.

In the algorithm, we have $\hat{M} \subseteq \hat{E}, p^{+}, p^{-} \in \mathbf{R}_{+}^{\hat{V}}$ and $z \in \mathbf{R}^{\mathcal{U}}$ in hand. We maintain that the characteristic vector of $\hat{M}$ is a feasible solution for $(\mathrm{P})$, and $\left(p^{+}, p^{-}, z\right)$ corresponds to a feasible solution $\left(y^{+}, y^{-}, z\right)$ for (D), where $y^{+}$and $y^{-}$are constructed by (10) and (11) from $p^{+}$and $p^{-}$, respectively. The goal of the algorithm is to achieve (6)-(9). Note that $c^{\prime}(e)=p^{+}\left(\partial^{+} e\right)+p^{-}\left(\partial^{-} e\right)+$ $\sum_{U \in \mathcal{U}, e \in E[U]} z(U)-c(e)$.

Here is a description of the algorithm, followed by detailed expositions of each procedure. In what follows, a pseudo-vertex into which $U \subseteq \hat{V}$ is shrunk is denoted by $v_{U}$. The $p^{+}$-minor of $\hat{\mathbf{M}}^{+}$ and $p^{-}$-minor of $\hat{\mathbf{M}}^{-}$are simply denoted by $\hat{\mathbf{M}}_{p}^{+}=\left(\hat{V}, \hat{\mathcal{I}}_{p}^{+}\right)$and $\hat{\mathbf{M}}_{p}^{-}=\left(\hat{V}, \hat{\mathcal{I}}_{p}^{-}\right)$, respectively. Similarly, $\mathbf{M}_{p}^{+}=\left(V, \mathcal{I}_{p}^{+}\right)$and $\mathbf{M}_{p}^{-}=\left(V, \mathcal{I}_{p}^{-}\right)$denote the matroids obtained by applying the procedures of shrinking of corresponding vertex subsets to $\hat{\mathbf{M}}_{p}^{+}$and $\hat{\mathbf{M}}_{p}^{-}$, respectively.

\section{Algorithm WIEF}

Input. An odd-cycle-symmetric weighted digraph $(\hat{G}, c)$ and matroids $\hat{\mathbf{M}}^{+}, \hat{\mathbf{M}}^{-}$.

Output. An independent even factor $\hat{M}$ in $\left(\hat{G}, \hat{\mathbf{M}}^{+}, \hat{\mathbf{M}}^{-}\right)$maximizing $c(\cdot)$, and $\left(p^{+}, p^{-}, z\right)$ corresponding to a dual optimal solution.

Step 1. Set $G=\hat{G}, \mathbf{M}^{+}=\hat{\mathbf{M}}^{+}, \mathbf{M}^{-}=\hat{\mathbf{M}}^{-}, \hat{M}=M=\emptyset, p^{+}(v)=\max \left\{c(e) \mid e \in \delta^{+} v\right\}$, $p^{-}(v)=0$ and $z(U)=0$.

Step 2. Construct an auxiliary digraph $G^{*}=\left(V^{*}, E^{*} ; S^{+}, S^{-}\right)$as follows:

$$
V^{*}=V^{+} \cup V^{-}, E^{*}=E^{\circ} \cup \bar{M} \cup J^{+} \cup J^{-},
$$


where

$$
\begin{aligned}
V^{+}= & \left\{v^{+} \mid v \in V\right\}, V^{-}=\left\{v^{-} \mid v \in V\right\}, \\
E^{\circ}= & \left\{u^{+} v^{-} \mid u v \in E \backslash M, c^{\prime}(e)=0\right\}, \\
\bar{M}= & \left\{v^{-} u^{+} \mid u v \in M\right\}, \\
J^{+}= & \left\{u^{+} v^{+} \mid u \in \partial^{+} M, v \in \operatorname{cl}_{p}^{+}\left(\partial^{+} M\right) \backslash \partial^{+} M, u \in C_{p}^{+}\left(\partial^{+} M \mid v\right)\right\}, \\
J^{-}= & \left\{u^{-} v^{-} \mid u \in \operatorname{cl}_{p}^{-}\left(\partial^{-} M\right) \backslash \partial^{-} M, v \in \partial^{-} M, v \in C_{p}^{-}\left(\partial^{-} M \mid u\right)\right\}, \\
S^{+}= & \left\{u^{+} \mid u \in V \backslash \mathrm{cl}_{p}^{+}\left(\partial^{+} M\right), p^{+}(u)>0\right\} \\
& \cup\left\{v_{U}^{+} \mid U \in \mathcal{U}, v_{U} \in V \backslash \mathrm{cl}_{p}^{+}\left(\partial^{+} M\right), p^{+}(u)>0 \text { for } u \in U \backslash \partial^{+} \hat{M}\right\}, \\
S^{-}= & \left\{v^{-} \mid v \in V \backslash \mathrm{cl}_{p}^{-}\left(\partial^{-} M\right)\right\} \cup\left\{u^{+} \mid u \in \partial^{+} M, p^{+}(u)=0\right\} \\
& \cup\left\{v_{U}^{+} \mid U \in \mathcal{U}, v_{U} \in \partial^{+} M, \exists v \in U, p^{+}(v)=0\right\} .
\end{aligned}
$$

If $S^{+}=\emptyset$, then return $\hat{M}$ and $\left(p^{+}, p^{-}, z\right)$, and halt.

Step 3. Let $R^{+} \subseteq V^{+}$and $R^{-} \subseteq V^{-}$be the sets of the reachable vertices from $S^{+}$. If $\left(R^{+} \cup\right.$ $\left.R^{-}\right) \cap S^{-}=\emptyset$, then go to Step 7 .

Step 4. Let $P^{*}$ be a path from $S^{+}$to $S^{-}$with minimum number of edges. Let $P \subseteq E$ be the edge set in $G$ which corresponds to $P^{*} \cap\left(E^{\circ} \cup \bar{M}\right)$. If $M \triangle P$ does not contain odd cycles, then apply Augment $(M, P)$, expand every pseudo-vertex $v_{U}$ with $z(U)=0$, and go to Step 2 .

Step 5. Let $C$ be an odd cycle which is to be shrunk if Shrink $(M, P)$ is applied. If there exists $v \in V(C)$ such that $v^{+} \in S^{-}$, then apply $\operatorname{Modify}(M, P)$, expand every pseudo-vertex $v_{U}$ with $z(U)=0$, and go to Step 2 .

Step 6. Apply Shrink $(M, P)$, and then go to Step 2.

Step 7. Apply Dual_Update, expand every pseudo-vertex $v_{U}$ such that $z(U)=0, v_{U}^{+} \notin R^{+}$and $v_{U}^{-} \in R^{-}$, and then go to Step 2.

\subsection{Step 2: auxiliary graph construction}

This subsection describes how to construct the auxiliary graph $G^{*}$ efficiently. The difficulty arises in testing independence in $\mathbf{M}^{+}$and $\mathbf{M}^{-}$using the independence oracle of $\hat{\mathbf{M}}^{+}$and $\hat{\mathbf{M}}^{-}$. The main idea is explained in [11], which is included below for completeness.

Consider how to determine $S^{+}$and $J^{+}$. We have $\partial^{+} M \in \mathcal{I}_{p}^{+}$and $\partial^{+} \hat{M} \in \hat{\mathcal{I}}_{p}^{+}$. Denote the set of pseudo-vertices $W=\left\{v_{U_{1}}, \ldots, v_{U_{k}}\right\}$. Now, construct another auxiliary graph $H_{p}^{+}=\left(\hat{V}, F_{p}^{+}\right)$ defined by

$$
\begin{aligned}
F_{p}^{+}=\left(\bigcup _ { i = 1 } ^ { k } \left\{u v \mid v_{U_{i}} \notin \partial^{+} M, u \in\right.\right. & \left.\left.U_{i} \cap \partial^{+} \hat{M}, v \in U_{i} \backslash \partial^{+} \hat{M}\right\}\right) \\
& \cup\left\{u v \mid u \in \hat{\operatorname{cl}}_{p}^{+}\left(\partial^{+} \hat{M}\right) \backslash \partial^{+} \hat{M}, v \in \partial^{+} \hat{M}, v \in \hat{C}_{p}^{+}\left(\partial^{+} \hat{M} \mid u\right)\right\} .
\end{aligned}
$$

Also, denote $T^{+}=\hat{V} \backslash \hat{c l}_{p}^{+}\left(\partial^{+} \hat{M}\right)$. 
For $v \in V \backslash \partial^{+} M$, we have to determine whether $v^{+} \in S^{+}$, and if not, enumerate edges in $J^{+}$ whose head is $v^{+}$. This can be done by a single search in $H_{p}^{+}$.

Assume that $v$ is not a pseudo-vertex. Then, $v^{+} \in S^{+}$if and only if there exists a path from $v$ to $T^{+}$in $H_{p}^{+}$. If such a path does not exist, then the edges in $J^{+}$whose head is $v$ are found as follows. For $u \in \partial^{+} M \backslash W, u^{+} v^{+} \in J^{+}$if and only if $H_{p}^{+}$has a path from $v$ to $u$. For $v_{U_{i}} \in \partial^{+} M$, $v_{U_{i}}^{+} v^{+} \in J^{+}$if and only if $H_{p}^{+}$has a path from $v$ to some vertex in $U_{i}$.

If $v$ is a pseudo-vertex $w_{j}$, in the above argument replace $v$ with the unique vertex in $U_{j} \backslash \partial^{+} \hat{M}$. All of these determinations can be done by decomposing $H_{p}^{+}$into strongly connected components.

Of course, the above argument applies to computing $S^{-}$and $J^{-}$.

\subsection{Steps 4-6: $\operatorname{Augment}(M, P), \operatorname{Modify}(M, P)$ and $\operatorname{Shrink}(M, P)$}

Let $P^{*}$ be a shortest path from $S^{+}$to $S^{-}$. Define $P \subseteq E$ by $P=\left\{u v \mid u^{+} v^{-} \in E^{\circ} \cap P^{*}\right\} \cup\{u v \mid$ $\left.v^{-} u^{+} \in \bar{M} \cap P^{*}\right\}$ and consider the edge set $M^{\prime}=M \triangle P$. Observe that $\left|\delta^{+} v \cap M^{\prime}\right| \leq 1$ and $\left|\delta^{-} v \cap M^{\prime}\right| \leq 1$ for every $v \in V$. Also, by the classical matroid intersection argument, it holds that $\partial^{+} M^{\prime} \in \mathcal{I}_{p}^{+}$and $\partial^{-} M^{\prime} \in \mathcal{I}_{p}^{-}$. Therefore, $M^{\prime}$ is a new independent even factor if $M^{\prime}$ contains no odd cycles, and an advantage is that $\left|S^{+}\right|$decreases. This procedure of obtaining $M^{\prime}$ is referred to as $\operatorname{Augment}(M, P)$.

Now, suppose $M^{\prime}$ contains odd cycles. Denote $P=\left\{e_{1}, m_{1}, e_{2}, \ldots, e_{l-1}, m_{l-1}, e_{l}\right\}$, where $e_{i} \in$ $E \backslash M, m_{i} \in M$ and the suffixes indicate the order of appearance in $P^{*}$. For $i=0,1, \ldots, l$, define a subset $P_{i}$ of $P$ by

$$
P_{i}= \begin{cases}\emptyset & (i=0), \\ \left\{e_{1}, m_{1}, e_{2}, m_{2}, \ldots, e_{i}, m_{i}\right\} & (i=1, \ldots, l-1) \\ P & (i=l),\end{cases}
$$

and let $i^{*}$ be the minimum integer $j$ such that $M \triangle P_{j}$ contains odd cycles. Note that $M \triangle P_{i^{*}}$ contains a unique odd cycle, denoted by $C$. Here, update $M$ by $M:=M \triangle P_{i^{*}-1}$. Note that this operation maintains $|M|$ and that $M$ is an independent even factor in $\left(G, \mathbf{M}_{p}^{+}, \mathbf{M}_{p}^{-}\right)$. Also, observe that $|E[V(C)] \cap M|=|C|-1$, that is, (9) holds for $U=V(C)$. Then, update $G$ by contracting $V(C)$ into a single vertex: identify all vertices in $V(C)$ to obtain a pseudo-vertex $w=v_{V(C)}$; and delete all edges in $E[V(C)]$. Namely, $G=(V, E)$ and $M \subseteq E$ are updated by

$$
\begin{aligned}
V:= & (V \backslash V(C)) \cup\{w\}, \\
E:= & \{u v \mid u, v \in V \backslash V(C), u v \in E\} \\
& \cup\{w v \mid v \in V \backslash V(C), \exists u \in V(C) \text { such that } u v \in E\} \\
& \cup\{u w \mid u \in V \backslash V(C), \exists v \in V(C) \text { such that } u v \in E\}, \\
M:= & \{u v \mid u, v \in V \backslash V(C), u v \in M\} \\
& \cup\{w v \mid v \in V \backslash V(C), \exists u \in V(C) \text { such that } u v \in M\} \\
& \cup\{u w \mid u \in V \backslash V(C), \exists v \in V(C) \text { such that } u v \in M\} .
\end{aligned}
$$

Finally, update $\mathbf{M}_{p}^{+}$and $\mathbf{M}_{p}^{-}$by applying the operation of shrinking of $V(C)$. This procedure of updating $G, M, \mathbf{M}_{p}^{+}$, and $\mathbf{M}_{p}^{-}$is referred to as $\operatorname{Shrink}(M, P)$. Note that parallel edges may appear after this procedure.

However, if $v^{+} \in S^{-}$for some $v \in V(C)$, we do not execute $\operatorname{Shrink}(M, P)$. Instead, after replacing $M$ with $M \triangle P_{i^{*}-1}$, we modify the edges of $M_{C}=M \cap C$. By odd-cycle-symmetry, $G^{*}$ also has $\bar{C}$, and there exists an edge set $M_{C}^{\prime} \subseteq C \cup \bar{C}$ such that $\partial^{+} M_{C}^{\prime}=V(C) \backslash\{v\}$ and $\partial^{-} M_{C}^{\prime}=\partial^{-} M_{C}$. 
Here, we update $M$ by $\left(M \backslash M_{C}\right) \cup M_{C}^{\prime}$. This procedure is referred to as $\operatorname{Modify}(M, P)$. Note that $\operatorname{Modify}(M, P)$ does not increase $|M|$, but decreases $\left|S^{+}\right|$as well as Augment $(M, P)$ does. Hence, in convenience we call Augment $(M, P)$ and $\operatorname{Modify}(M, P)$ collectively as an augmentation. Also, $\operatorname{Augment}(M, P)$, Modify $(M, P)$ and $\operatorname{Shrink}(M, P)$ are collectively referred to as a primal change.

Proposition 7. In primal changes, it is maintained that $M$ is an independent even factor in $\left(G, \mathbf{M}_{p}^{+}, \mathbf{M}_{p}^{-}\right)$.

Finally, let us explain how to expand pseudo-vertices. Let $w \in V$ be a pseudo-vertex which is to be expanded to a vertex set $U$. Denote the corresponding odd cycle by $C$. Expanding of $w$ is the reverse operation of shrinking $U$ : replace $w$ with $U$; reconstruct the edges in $E[U]$; reconnect the edges in $\delta^{+} w$ and $\delta^{-} w$ to a vertex in $U$ to which it was incident before shrinking $U$. The problem is to decide which of the $|U|-1$ edges in $C \cup \bar{C}$ to put into $M$. If $M$ has two edges $u w_{+}$and $w_{-} v$ such that $w_{+}, w_{-} \in U$ and $u, v \notin U$, then choose the edges in $C \cup \bar{C}$ that form an even path $P_{U}$ from $w_{+}$to $w_{-}$and vertex-disjoint cycles of length two that cover the vertices in $U \backslash V\left(P_{U}\right)$. If $u w_{+}$and $w_{-} v$ does not exist, we know which vertex should not be in $\partial^{+} M$ or $\partial^{-} M$ by $\partial^{+} \hat{M}$ and $\partial^{-} \hat{M}$. Then, consider such a vertex as $w_{+}$or $w_{-}$, and apply the above procedure.

Remark 8. In primal changes, we also update $\hat{M}$, which can be done by tracing the paths in $H_{p}^{+}$ or $H_{p}^{-}$corresponding to the edges in $P^{*} \cap\left(J^{+} \cup J^{-}\right)$.

Remark 9. By Shrink $(M, P)$, the initial vertex of $P$ leaves $S^{+}$, and the newly created pseudovertex $w$ belongs to $S^{+}$. Thus, in contrast to classical weighted matching and matroid intersection algorithms, $\partial^{+} \hat{M}$ does not maximize $p^{+}\left(\partial^{+} \hat{M}\right)$ among the independent sets with the same size as $\partial^{+} \hat{M}$. Instead of keeping $\partial^{+} \hat{M}$ to maximize $p^{+}\left(\partial^{+} \hat{M}\right)$, we have that there exists an independent set $I \in \hat{\mathcal{I}}^{+}$maximizing $p^{+}(I)$ such that contains $\partial^{+} \hat{M}$, i.e., $\partial^{+} \hat{M} \in \hat{\mathcal{I}}_{p}^{+}$. This is also the reason why we have $S^{-}$in the $V^{+}$-side.

We have another reason why we introduced the notion of $p$-minor. In auxiliary graphs in classical weighted matroid intersection algorithms, we have sets of edges connecting exchangeable vertices in $\mathbf{M}^{+}$(resp., $\mathbf{M}^{-}$) with the same value of $p^{+}$(resp., $p^{-}$). In our algorithm, $p^{+}$and $p^{-}$are not defined on the pseudo-vertices, and so it is difficult to define $J^{+}$(resp., $J^{-}$) by the exchangeability in $\mathbf{M}^{+}$(resp., $\mathbf{M}^{-}$) and the value of $p^{+}$(resp., $p^{-}$). Instead, we define $J^{+}$(resp., $J^{-}$) by the exchangeability in $\mathbf{M}_{p}^{+}$(resp., $\mathbf{M}_{p}^{-}$).

Remark 10. In the procedures of $\operatorname{Modify}(M, P)$ and expanding a pseudo-vertex, we require that the odd cycle $C$ has a reverse cycle $\bar{C}$ and $c^{\prime}(e)=0$ for every edge in $\bar{C}$, which is certified by the following propositions.

Proposition 11 (Pap [20]). The procedure Shrink $(M, P)$ preserves the odd-cycle-symmetry of $G$.

Proposition 12 (Takazawa [24]). If $c^{\prime}(e)=0$ for every edge $e$ in an odd cycle $C$, then $c^{\prime}(e)=0$ for every edge e in $\bar{C}$.

\subsection{Step 7: Dual_Update}

Associated with $R^{+}$and $R^{-}$, define $\hat{R}^{+}, \hat{R}^{-} \subseteq \hat{V}$ by

$$
\begin{aligned}
& \hat{R}^{+}=\left\{v \in \hat{V} \mid v^{+} \in R^{+} \text {or } v \in U \text { for some pseudo-vertex } v_{U} \text { s.t. } v_{U}^{+} \in R^{+}\right\}, \\
& \hat{R}^{-}=\left\{v \in \hat{V} \mid v^{-} \in R^{-} \text {or } v \in U \text { for some pseudo-vertex } v_{U} \text { s.t. } v_{U}^{-} \in R^{-}\right\} .
\end{aligned}
$$


In Dual_Update, we update the dual variables by

$$
\begin{aligned}
p^{+}(v) & := \begin{cases}p^{+}(v)-\epsilon & \left(v \in \hat{R}^{+}\right), \\
p^{+}(v) & (\text { otherwise }),\end{cases} \\
p^{-}(v) & := \begin{cases}p^{-}(v)+\epsilon & \left(v \in \hat{R}^{-}\right), \\
p^{-}(v) & (\text { otherwise }),\end{cases} \\
z(U): & = \begin{cases}z(U)+\epsilon & \left(U \in \mathcal{U}, v_{U}^{+} \in R^{+}, v_{U}^{-} \notin R^{-}\right), \\
z(U)-\epsilon & \left(U \in \mathcal{U}, v_{U}^{+} \notin R^{+}, v_{U}^{-} \in R^{-}\right), \\
z(U) & \text { (otherwise), }\end{cases}
\end{aligned}
$$

where

$$
\begin{aligned}
& \epsilon=\min \left\{\epsilon_{1}, \epsilon_{2}, \epsilon_{3}, \epsilon_{4}, \epsilon_{5}\right\}, \\
& \epsilon_{1}=\min \left\{p^{+}(v) \mid v \in \hat{R}^{+}\right\}, \\
& \epsilon_{2}=\min \left\{z(U) \mid U \in \mathcal{U}, v_{U}^{+} \notin R^{+}, v_{U}^{-} \in R^{-}\right\}, \\
& \epsilon_{3}=\min \left\{c^{\prime}(e) \mid e \in \hat{E}, \partial^{+} e \in \hat{R}^{+}, \partial^{+} e \notin \hat{R}^{-}\right\}, \\
& \epsilon_{4}=\left\{p^{+}(u)-p^{+}(v) \mid u \in \hat{R}^{+}, v \notin \hat{R}^{+}, u \in C^{+}\left(\hat{B}_{p}^{+} \mid v\right)\right\}, \\
& \epsilon_{5}=\left\{p^{-}(v)-p^{-}(u) \mid u \in \hat{R}^{-}, v \notin \hat{R}^{-}, v \in C^{-}\left(\hat{B}_{p}^{-} \mid u\right)\right\} .
\end{aligned}
$$

Here, $\hat{B}_{p}^{+}$is a base of $\hat{\mathbf{M}}_{p}^{+}$containing $\partial^{+} \hat{M}$, and $\hat{B}_{p}^{-}$is a base of $\hat{\mathbf{M}}_{p}^{-}$containing $\partial^{-} \hat{M}$.

Let us see that Dual_Update maintains the feasibility of the dual solution.

Proposition 13. In Algorithm WIEF, the dual variables are feasible for (D).

Proof. Note that the dual variables are feasible for (D) in the beginning of the algorithm.

Consider (5). For an edge $e \in \hat{E}$, observe that $c^{\prime}(e)$ changes in Dual_Update only if $e$ is not shrunk, $\partial^{+} e \in \hat{R}^{+}$and $\partial^{-} e \notin \hat{R}^{-}$. In this case $c^{\prime}(e)$ decreases by $\epsilon$, and is still nonnegative because $\epsilon \leq \epsilon_{3}$.

For (2) and (3), $y^{+} \geq 0$ holds if $p^{+} \geq 0$, and $y^{-} \geq 0$ if $p^{-} \geq 0$ by (10) and (11). The nonnegativity of $p^{+}$follows from $\epsilon \leq \epsilon_{1}$, and that of $p^{-}$is easy because $p^{-}$is monotonically nondecreasing.

Finally, (4) follows from $\epsilon \leq \epsilon_{2}$.

Moreover, by the following proposition, we also claim that $M$ is still an independent even factor in $\left(G, \mathbf{M}_{p}^{+}, \mathbf{M}_{p}^{-}\right)$after Dual_Update.

Proposition 14. After Dual_Update, $M$ is an independent even factor in $\left(G, \mathbf{M}_{p}^{+}, \mathbf{M}_{p}^{-}\right)$.

Proof. It suffices to prove that $\hat{B}_{p}^{+} \in \hat{\mathcal{I}}_{p}^{+}$and $\hat{B}_{p}^{-} \in \hat{\mathcal{I}}_{p}^{-}$are maintained. For $\hat{B}_{p}^{+}$, it holds that $p^{+}(u) \geq p^{+}(v)$ for every pair of $u$ and $v$ such that $u \in C^{+}\left(\hat{B}_{p}^{+} \mid v\right)$, which is maintained in Dual_Update since $\epsilon \geq \epsilon_{4}$. The same argument applies to $\hat{B}_{p}^{-}$.

\subsection{Complexity analysis}

So far, we have seen that Algorithm WIEF maintains primal and dual feasible solutions. What is left to show is that they become optimal in polynomial time. Recall that $|\hat{V}|=n,|\hat{E}|=m$, and $\gamma$ is the time for an independence test.

First, let us see that CS conditions (6), (8) and (9) are maintained. Observe that these three conditions hold in the beginning of the algorithm. 
Condition (6). Suppose $e$ comes to be in $M$ in a primal change. Since a primal change is executed in $G^{*}$, we have $e \in E^{\circ}$, that is, $c^{\prime}(e)=0$ holds. Afterwards, in a subsequent Dual_Update, it holds that both of $\left(\partial^{+} e\right)^{+}$and $\left(\partial^{-} e\right)^{-}$are reachable from $S^{+}$, or both of them are not. Hence, $c^{\prime}(e)$ does not change in Dual_Update.

Consider expanding of an odd cycle $C$. We have that $c^{\prime}(e)=0$ for an edge $e$ in $C$ when $C$ was shrunk, and the same holds for $\bar{C}$ by odd-cycle-symmetry. Moreover, observe that $c^{\prime}(e)$ does not change in Dual_Update for a shrunk edge $e$. Therefore, in expanding $C$, it holds that $c^{\prime}(e)=0$ for every edge $e \in C \cup \bar{C}$.

Condition (8). By Proposition 6, it suffices to show that $p^{-}(v)=0$ for $v \in \hat{V} \backslash \hat{\mathrm{cl}}_{p}^{-}\left(\partial^{-} \hat{M}\right)$. This can be proved through the fact that $p^{-}(v)$ is changed only if $v \in \hat{R}^{-} \subseteq \hat{\mathrm{cl}}_{p}^{-}\left(\partial^{-} \hat{M}\right)$ and $\hat{\mathrm{cl}}_{p}^{-}\left(\partial^{-} \hat{M}\right)$ is monotonically nondecreasing.

Condition (9). We have that $z(U)>0$ only if $U$ is shrunk. For such $U$, the procedure of expanding suggests that $x(E[U])=|U|-1$.

Next, consider how many times each procedure is executed until (7) holds.

Proposition 15. The procedures of $\operatorname{Augment}(M, P)$ and $\operatorname{Modify}(M, P)$ respectively happen at most $n$ times throughout Algorithm WIEF.

Proof. The procedure Augment $(M, P)$ increases $|\hat{M}|$ by one, and $|\hat{M}|$ never decreases in Algorithm WIEF.

In $\operatorname{Modify}(M, P)$, a vertex $v \in \hat{V}$ comes to satisfy $v \notin \partial^{+} \hat{M}$ and $p^{+}(v)=0$. Once these two conditions are satisfied at a vertex, they remain satisfied, and Algorithm WIEF terminates if they are satisfied at every vertex.

Proposition 16. The procedure Shrink $(M, P)$ happen $\mathrm{O}(n)$ times between augmentations.

Proof. In Shrink $(M, P),|V|$ decreases by at least two. Hence, in order to prove this proposition, it suffice to show that a pseudo-vertex $w$ created after an augmentation is not expanded until the next augmentation.

When $w$ is created, $w^{+} \in S^{+}$holds, and hence $w^{+} \in R^{+}$. This is maintained in the subsequent procedures of Shrink $(M, P)$ and Dual_Update until the next augmentation. Since a pseudo-vertex $w$ is expanded after Dual_Update only if $w^{+} \notin R^{+}$and $w^{-} \in R^{-}, w$ is not expanded until the next augmentation.

Proposition 17. It holds that $\epsilon>0$ in Dual_Update, and Dual_Update happen $\mathrm{O}(n)$ times between primal changes.

Proof. Suppose $\epsilon=\epsilon_{1}=p^{+}(v)$ for $v \in \hat{R}^{+}$. If $p^{+}(v)=0$, then, it implies that a path from $S^{+}$to $S^{-}$exists, and thus a primal change is executed instead of Dual_Update. Thus, $\epsilon_{1}>0$ and a primal change follows a Dual_Update with $\epsilon=\epsilon_{1}$.

Suppose $\epsilon=\epsilon_{2}=z(U)$ for a pseudo-vertex $v_{U}$ with $v_{U}^{+} \notin R^{+}$and $v_{U}^{-} \in R^{-}$. Since a pseudovertex $w$ created after the latest augmentation satisfies that $w^{+} \in R^{+}$, we have that $v_{U}$ was created before the latest augmentation. As $v_{U}$ was not expanded after the augmentation, $z(U)>0$ at the augmentation. Moreover, by the rule of expanding of pseudo-vertices after Dual_Update, we conclude that $z(U)>0$ at this Dual_Update. Also, the number of times of $\epsilon=\epsilon_{2}$ is bounded by $n$.

Suppose $\epsilon=\epsilon_{3}, \epsilon_{4}$, or $\epsilon_{5}$. In such a case, an edge $e$ comes to be in $G^{*}$ and $\partial^{-} e$ becomes reachable from $S^{+}$. Since $\hat{R}^{+}$and $\hat{R}^{-}$are nondecreasing between augmentations, the number of times of $\epsilon$ hits $\epsilon_{3}, \epsilon_{4}$ and $\epsilon_{5}$ is $\mathrm{O}(n)$ between primal changes. 
By the above propositions, we execute $\mathrm{O}\left(n^{2}\right)$ times of primal changes and $\mathrm{O}\left(n^{3}\right)$ times of Dual_Update until (7) holds. After a primal change, we reconstruct the auxiliary graph $G^{*}$, which can be done in $\mathrm{O}\left(n^{2} \gamma\right)$ time (see Section 4.1). On the other hand, a single iteration of Dual_Update takes $\mathrm{O}\left(n^{2}\right)$ time. Therefore, the total time complexity of Algorithm WIEF is $\mathrm{O}\left(n^{4} \gamma+n^{5}\right)$.

Theorem 18. Algorithm WIEF runs in $\mathrm{O}\left(n^{4} \gamma+n^{5}\right)$ time.

For the special case where the given matroids are linear, reconstruction of $G^{*}$ takes $\mathrm{O}\left(n^{3}\right)$ time. Thus, the time complexity of Algorithm WIEF is $\mathrm{O}\left(n^{5}\right)$, while that of Cunningham and Geelen's method for this case is $\mathrm{O}\left(n^{6} m\right)$.

Theorem 19. If linear representations of the matroids are given, Algorithm WIEF runs in $\mathrm{O}\left(n^{5}\right)$ time.

\subsection{Proof for Theorem 4}

We have seen that Algorithm WIEF finds a pair of optimal solution $x$ of $(\mathrm{P})$ and $\left(y^{+}, y^{-}, z\right)$ of (D) for an odd-cycle-symmetric weighted digraph $(G, c)$. Obviously $x \in\{0,1\}^{E}$. Moreover, since Dual_Update only consists of addition, subtraction and comparison, $\left(y^{+}, y^{-}, z\right)$ is also integer if $c$ is integer. Therefore, Theorem 4 holds.

\section{Acknowledgements}

The author is thankful to Satoru Iwata for discussions and comments on this topic. The author also thanks Nicholas J. A. Harvey for his comments at an early stage of this work. This work is supported by Grant-in-Aid for Scientific Research.

\section{References}

[1] Bouchet, A., Cunningham, W.H.: Delta-matroids, jump systems, and bisubmodular polyhedra. SIAM J. Discrete Math. 8, 17-32 (1995)

[2] Cunningham, W.H.: Matching, matroids, and extensions. Math. Program. 91, 515-542 (2002)

[3] Cunningham, W.H., Geelen, J.F.: The optimal path-matching problem. Combinatorica 17, 315-337 (1997)

[4] Cunningham, W.H., Marsh, III, A.B.: A primal algorithm for optimum matching. Math. Program. Study 8, 50-72 (1978)

[5] Edmonds, J.: Maximum matching and a polyhedron with 0,1-vertices. J. Res. Natl. Bur. Stand. Sect. B 69, 125-130 (1965)

[6] Edmonds, J.: Paths, trees, and flowers. Can. J. Math. 17, 449-467 (1965)

[7] Edmonds, J.: Submodular functions, matroids, and certain polyhedra. In: Guy, R., Hanani, H., Sauer, N., Schönheim, J. (eds.) Combinatorial Structures and Their Applications, pp. 69-87, Gordon and Breach, New York (1970)

[8] Edmonds, J.: Matroid intersection. Ann. Discrete Math. 4, 39-49 (1979) 
[9] Frank, A., Szegő, L.: Note on the path-matching formula. J. Graph Theory 41, 110-119 (2002)

[10] Harvey, N.J.A.: Algebraic structures and algorithms for matching and matroid problems. In: Proceedings of the 47th Annual IEEE Symposium on Foundations of Computer Science, pp. $531-542(2006)$

[11] Iwata, S., Takazawa, K.: The independent even factor problem. SIAM J. Discrete Math. 22, 1411-1427 (2008)

[12] Király, T., Makai, M.: On polyhedra related to even factors. In: Bienstock, D., Nemhauser, G.L. (eds.) Integer Programming and Combinatorial Optimization: Proceedings of the 10th International IPCO Conference, Lecture Notes on Computer Science 3064, pp. 416-430, Springer-Verlag, Heidelberg (2004)

[13] Kobayashi, Y., Takazawa, K.: Even factors, jump systems, and discrete convexity. J. Comb. Theory Ser. B 99, 139-161 (2009)

[14] Lawler, E.L.: Matroid intersection algorithms. Math. Program. 9, 31-56 (1975)

[15] Murota, K.: Valuated matroid intersection I: optimality criteria. SIAM J. Discrete Math. 9, $545-561(1996)$

[16] Murota, K.: Valuated matroid intersection II: algorithms. SIAM J. Discrete Math. 9, 562-576 (1996)

[17] Murota, K.: M-convex functions on jump systems: a general framework for minsquare graph factor problem. SIAM J. Discrete Math. 20, 231-226 (2006)

[18] Oxley, J.G.: Matroid Theory. Oxford University Press, Oxford (1992)

[19] Pap, G.: A Constructive Approach to Matching and Its Generalizations. Ph.D. thesis, Eötvös Loránd University (2006)

[20] Pap, G.: Combinatorial algorithms for matchings, even factors and square-free 2-factors. Math. Program. 110, 57-69 (2007)

[21] Pap, G., Szegö, L.: On the maximum even factor in weakly symmetric graphs. J. Comb. Theory Ser. B 91, 201-213 (2004)

[22] Spille, B., Szegö, L.: A Gallai-Edmonds-type structure theorem for path-matchings. J. Graph Theory 46, 93-102 (2004)

[23] Spille, B., Weismantel, R.: A generalization of Edmonds' matching and matroid intersection algorithms. In: Cook, W.J., Schulz, A.S. (eds.) Integer Programming and Combinatorial Optimization: Proceedings of the 9th International IPCO Conference, Lecture Notes in Computer Science 2337, pp. 9-20, Springer-Verlag, Heidelberg (2002)

[24] Takazawa, K.: A weighted even factor algorithm. Math. Program. 115, 223-237 (2008) 